\title{
SENSITIVITY ENHANCEMENT USING PARAMETRIC AMPLIFICATION IN A RESONANT SENSING ARRAY
}

\author{
Z. Yie ${ }^{1 *}$, K.L. Turner ${ }^{1}$, N.J. Miller ${ }^{2}$, and S.W. Shaw ${ }^{2}$ \\ ${ }^{1}$ University of California-Santa Barbara, Santa Barbara, California, USA \\ ${ }^{2}$ Michigan State University, East Lansing, Michigan, USA
}

\begin{abstract}
In this work we describe the parametric amplification of a multi-degree of freedom resonant chemical mass sensing array. We experimentally demonstrate that parametric forcing can be employed to increase the effective resonant quality factor of the array structure via an applied base motion containing the appropriate frequency content. Applying parametric forcing in this manner is simple and aligns naturally with the vibrational properties of the sensing structure. Our effort to increase the effective quality factor is motivated by the desire to enhance the sensitivity of the device.
\end{abstract}

\section{INTRODUCTION}

Resonant chemical mass sensing using N/MEMS has received a great deal of attention over recent years [1]. The fundamental factors limiting sensitivity have largely been identified [2] and the question remains how best to manipulate them. In the pursuit of higher mass sensitivity, in this work we experimentally demonstrate parametric amplification, first demonstrated in MEMS in [3], as a means to improve the resonant quality factors, $Q$, of multiple resonances in a sensor array.

It has been shown that minimum detectable mass scales with the quality factor according to $Q^{-1 / 2}$ [4]. Thus, increasing $Q$ can be an effective way of reducing the minimum detectable mass and hence increase device sensitivity. However, in many environments, and for typical systems, $Q$ is limited by intrinsic dissipation. Numerous researchers have employed various techniques to reduce dissipation effects. Some have inverted the sensor by placing the dissipative environment inside microchannels embedded in the device while leaving vacuum outside [5]. Although this method is effective, the device fabrication complexity is significantly increased. Others have used feedback control methods to reduce the effect of dissipation, for example in $[6,7]$, where feedback was used to boost the effective quality factor of a single MEMS resonator. However, in order to apply feedback control, a means of detecting the device behavior is necessary. For a sensor array, this means that the behavior of each sensor element in the array must be known, a requirement that also increases device complexity.

Parametric amplification, an example of vibrational control [8], is an open-loop control strategy that can be easily implemented on an entire MEMS sensor array without the need for any feedback to monitor the device behavior. The only thing required is to develop a method for parametrically forcing the array. Moreover, it can be implemented in cooperation with a concurrent frequency sweep [9] to increase the quality factor of any resonance encountered by the frequency sweep without prior knowledge of the resonance location. This makes parametric amplification an approach worth consideration for quality factor control in swept systems. This is also of particular importance in this work because our sensor array is a multi-degree of freedom resonator and, as such, lacks the guaranteed 90 degrees phase lag at resonance found in single-degree of freedom resonators. Accordingly our device employs a frequency sweep for resonance tracking. The primary deficiency of this method, however, is that it is not effective for devices with significant inherent damping. Consequently, we have yet to demonstrate the effect at atmospheric pressure.

The sensor array of interest is the single-input-single-output scheme proposed in [10] for the simultaneous detection of multiple analytes. The device is shown in Fig. 1 and composed of four slightly detuned microcantilevers $\mathrm{M}_{1}, \mathrm{M}_{2}, \mathrm{M}_{3}$, and $\mathrm{M}_{4}$, attached to a common oscillator comprised of a cantilever plate SM. The sensor is forced via base motion applied to the entire structure and the response is monitored at a single point (labeled L) on the shuttle cantilever.

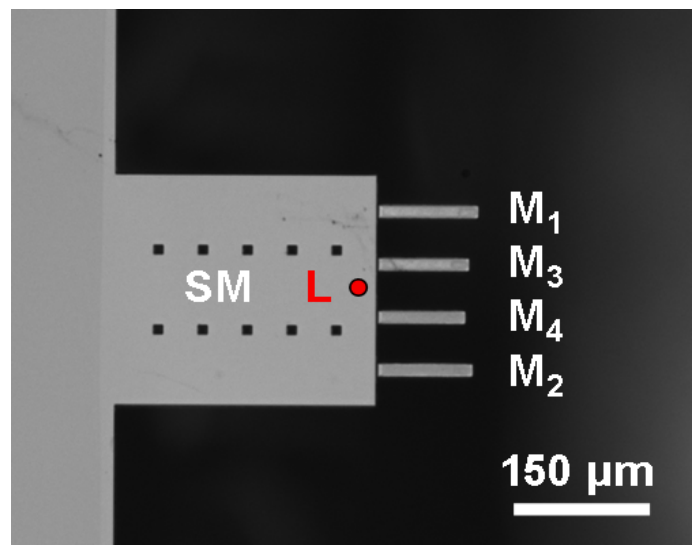

Figure 1: Microscope image depicting the sensor array with labeled shuttle cantilever $S M$, microcantilevers $M_{1}$ through $M_{4}$, and detection location $L$.
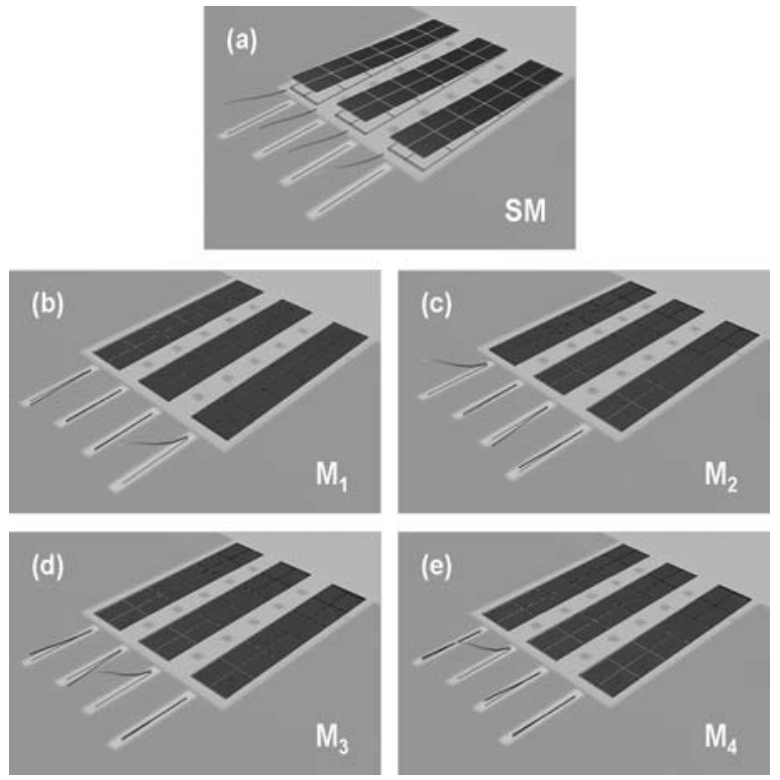

Figure 2: Experimentally measured mode shapes of the device. The mode shape in (a) corresponds to the shuttle cantilever SM. The mode shapes in (b)-(e) correspond to microcantilevers $M_{1}$ through $M_{4}$, and are physically localized. 
The modes of interest for this device are physically localized in the individual microcantilevers (see Fig. 2). These are intentionally designed to correlate frequency response features, specifically, the location of resonance peaks, with mass attachment sites in the array. The attachment sites are the tips of the microcantilevers. A frequency sweep is used to monitor the level of attached mass at each site. By piggybacking parametric excitation, also known as parametric pumping, on the frequency sweep, at twice the basic sweep frequency and with a fixed relative phase, we can effectively amplify the important resonances of the sensing structure. This can be done simply by altering the direction of the base motion relative to the plane of the device and adding a second harmonic term to the base motion signal with an appropriate phase $[9,11]$.

\section{THEORY}

In principle, the vibrations of the device are described by a partial differential equation. By projecting onto the four modes of interest, we can describe the system with a lumped parameter model

$$
\ddot{x}+Z \dot{x}+\Omega x+\Delta(t) x=F(t)
$$

where $x$ is a 4-vector describing the component of vibration in each of the four modes of interest, $\Omega$ is a diagonal matrix whose elements are the squared natural frequencies for each mode, $Z$ is the modal dissipation matrix, $F(t)$ is the forcing vector, and $\Delta(t)$ is the parametric forcing matrix. $Z$ is assumed to be small and diagonal, while $F(t)$ and $\Delta(t)$ are also assumed to be small and periodic in time.

Equation (1) describes four linear vibration modes coupled through parametric forcing. In our system, the parametric forcing arises from the base motion-induced inertial force along the axis of the device. In cantilevered structures, this force has been shown to result in parametric excitation [11]. For our analysis, we assume that $F(t)$ and $\Delta(t)$ take the form

$$
F(t)=f \cos (\omega t+\phi), \Delta(t)=\delta \cos (2 \omega t),
$$

where $f$ and $\delta$ are a vector and constant matrix, respectively. In principle, $F(t)$ and $\Delta(t)$ should contain both harmonics since they both arise from the same base motion. However, we have only kept the terms that produce resonant responses. The phase difference $\phi$, between $F(t)$ and $\Delta(t)$ can be chosen to produce the maximum quality factor amplification. The quality factor can also be attenuated if the phase relationship is chosen differently [11].

In order to have a clean parametric amplification of the modes of interest, it is necessary for them to have a degree of frequency isolation from other modes. In particular, if the modes of interest span a frequency band of $\left[\omega_{1}, \omega_{4}\right]$, then there can be no resonances in the frequency bands $\left[2 \omega_{1}, 2 \omega_{4}\right]$ or $\left[3 \omega_{1}, 3 \omega_{4}\right]$. Moreover, the condition for two modes with frequencies $\omega_{i}$ and $\omega_{j}$ to couple though parametric forcing is

$$
\omega \approx\left|\omega_{i} \pm \omega_{j}\right| \text { or } 2 \omega \approx\left|\omega_{i} \pm \omega_{j}\right|
$$

and so the device should have $2 \omega_{1}>\omega_{4}$. If these conditions hold, then the pump-strength/pump-frequency parameter space will have several Arnold tongues corresponding to the resonances, $\omega=\omega_{i}$, and combination resonances $2 \omega=\omega_{i}+\omega_{j}$. Inside these tongues, the linear system will become unstable and exhibit parametric resonance. However, if we maintain the pump strength below these tongues and sweep the direct and parametric forcing frequencies together (i.e. sweep $\omega$ ), then the system will remain stable and the quality factor of the resonances encountered during the sweep will be increased [9]. Furthermore, the combination resonances will not show up in the response because they will not be simultaneously forced directly and parametrically with resonant forcing [9]. This is because of the locked frequency relationship between the direct and parametric forcing. The stability requirement is given by [12]

$$
\delta_{i j} \delta_{j i}<4 Z_{i i} Z_{j j} \omega_{i} \omega_{j} \quad \forall i, j
$$

The maximal effective quality factor for a resonance, at frequency $\omega_{i}$, encountered during the sweep is

$$
Q_{e f f}=\frac{\omega_{i}^{2}}{\sigma_{1 / 2}},
$$

where $\sigma_{1 / 2}$ is the frequency detuning at half power, given by

$$
\begin{aligned}
& \sigma_{1 / 2}=\frac{1}{2} \sqrt{(\alpha-1)\left(-2+\sqrt{4+(\alpha+1)^{2}}\right)}, \\
& \alpha=\frac{2 Z_{i i} \omega_{i}}{\delta_{i i}} .
\end{aligned}
$$

The quality factor can be increased as $\alpha$ approaches 1 so long as the amplitude of the response remains sufficiently small so that nonlinear effects do not come into play. When this happens, the gain in resonance quality is limited [13].

\section{RESULTS}

In experiment, the base motion is provided by an externally mounted shear-type piezo-actuator (P-121.01, Physik Instrumente). The frequency response of each microcantilever is captured from a single point measurement on the shuttle cantilever using a laser vibrometer (see Fig. 1). The shuttle cantilever has a measured resonant frequency of $50.55 \mathrm{kHz}$, while the resonant frequencies of the microcantilevers range from $71-93 \mathrm{kHz}$. For all experiments, the device was operated in a test chamber at 50 mTorr.

Depending on the applied driving signal, the shear piezo provides movements in both the out-of-plane and in-plane directions, which can be used to realize both direct and parametric forcing. By applying a small driving voltage, enough out-of-plane motions can be created to achieve direct forcing. Applying a larger driving voltage allows the shear piezo to generate strong in-plane motions and achieve parametric forcing. To implement parametric amplification, we set the piezo-forcing function to be

$$
A_{d} \cos (\omega t+\phi)+A_{p} \cos (2 \omega t)
$$

where $A_{d}$ represents the peak voltage amplitude for the effective direct forcing and $A_{p}$ presents the peak voltage amplitude for the effective parametric forcing. The phase difference $\phi$, between the two forcing terms was set to $90^{\circ}$ for a first run. The theoretical optimal phase is $45^{\circ}$, but due to experimental difficulties we were unable to vary the phase.

In order to determine the appropriate values for $A_{p}$, we first map the Arnold tongues for each microcantilever. Fig. 3 depicts the experimental curves. These plots were obtained by setting the piezo-driving frequency $\omega$ to be close to twice the resonant frequencies of the microcantilevers and then gradually increasing the driving voltage until a significant response was observed at half the driving frequency. From these plots, the minimum threshold 
voltage $V_{\text {th }}$ necessary for parametric resonance for each microcantilever can be obtained. Note that we were unable to locate the combination resonances because the effective pump strength at the combination resonances is extremely weak. This implies that the matrix $\delta$ is approximately diagonal. While this is not generally true, the localized modes of our device make this possible. This arises because the inner product of any two nonidentical modes with respect to the differential operator in the governing partial differential equation of this system is approximately zero as a result of the localization. Accordingly, parametric amplification not only allows for the increase in the effective quality factor, but does so without introducing spurious frequency response features such as combination resonances.
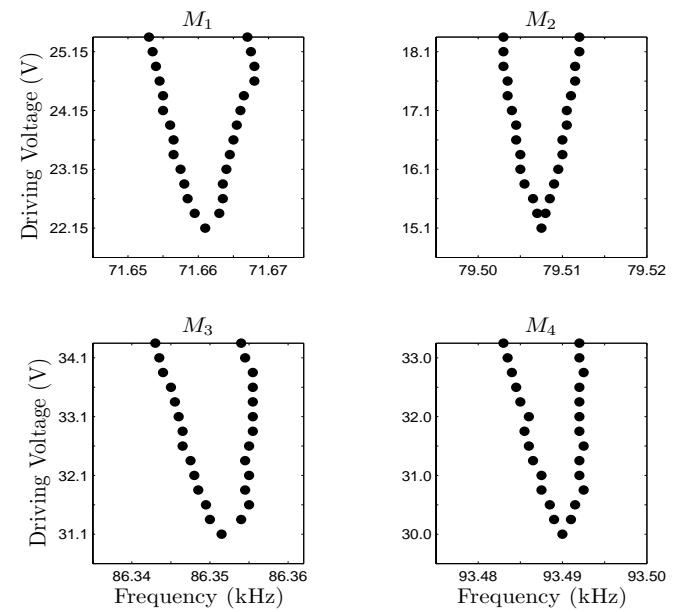

Figure 3: Experimental mapping of the Arnold tongues for microcantilevers $M_{1}$ through $M_{4}$.

Table 1 shows the value of $A_{p}$ for each microcantilever which was selected to be below the corresponding threshold voltage. The value of $A_{d}$ was set to $0.05 \mathrm{~V}$, which is small enough in amplitude to ensure that neither parametric resonance nor nonlinearity effects can be induced.

Table 1: Resonant frequency $\omega_{0}$, threshold voltage $V_{\text {th }}$, and parametric forcing amplitude $A_{p}$ for microcantilevers $M_{1}$ through $M_{4}$.

\begin{tabular}{l|c|c|c}
\hline & $\omega_{0}(\mathrm{kHz})$ & $V_{\text {th }}(\mathrm{V})$ & $A_{p}(\mathrm{~V})$ \\
\hline $\mathrm{M}_{1}$ & 71.66 & 22.15 & 21.3 \\
$\mathrm{M}_{2}$ & 79.50 & 15.1 & 14.25 \\
$\mathrm{M}_{3}$ & 86.35 & 31.1 & 30.25 \\
$\mathrm{M}_{4}$ & 93.49 & 30.0 & 27.5 \\
\hline
\end{tabular}

To show parametric amplification and to characterize the parametric gain, we first perform direct forcing on each of the microcantilevers by actuating the shear piezo with a $0.05 \mathrm{~V}$ sinusoidal driving signal around their resonant frequencies. We then add the parametric forcing term by implementing the input given in equation (8). The plots in Fig. 4 show the frequency response of the microcantilevers with and without parametric forcing. The resonances clearly exhibit an enhancement of the effective resonant quality factor values.

All the frequency responses were curve-fitted to obtain the effective resonant quality factor values before and after parametric amplification. A parametric gain of two or more was calculated for all the microcantilevers as summarized in Table 2 .
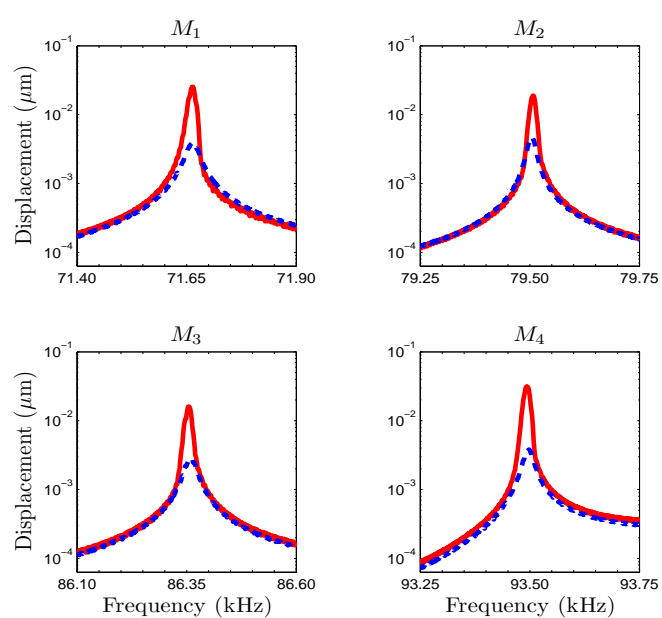

Figure 4: Frequency response of microcantilevers $M_{1}$ through $M_{4}$. The blue dashed line plots indicate direct forcing only, while the red solid line plots indicate the addition of parametric forcing.

As an illustration of the practical operation of the sensor, a full range frequency sweep was performed between 71 and $94 \mathrm{kHz}$ with and without parametric amplification. This is shown in Fig. 5, where the value of $A_{p}$ was set to $14.25 \mathrm{~V}$ to give $\mathrm{M}_{2}$ the maximum amplification without inducing instability.

Table 2: Effective $Q$ values for microcantilevers $M_{1}$ through $M_{4}$ before and after parametric amplification.

\begin{tabular}{c|c|c|c}
\hline & \multicolumn{3}{|c}{ Effective Q } \\
\cline { 2 - 4 } & $\begin{array}{c}\text { Parametric } \\
\text { Amplification } \\
\text { off }\end{array}$ & $\begin{array}{c}\text { Parametric } \\
\text { Amplification } \\
\text { on }\end{array}$ & Gain \\
& $Q_{\text {off }}$ & $Q_{\text {on }}$ & \\
\hline $\mathrm{M}_{1}$ & 1409 & 3751 & 2.6 \\
$\mathrm{M}_{2}$ & 2539 & 5080 & 2.0 \\
$\mathrm{M}_{3}$ & 1894 & 4944 & 2.6 \\
$\mathrm{M}_{4}$ & 2407 & 5604 & 2.3 \\
\hline
\end{tabular}

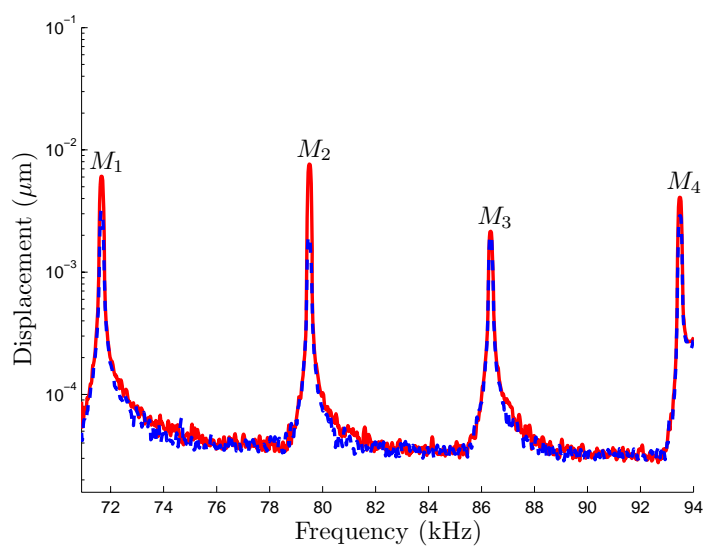

Figure 5: Full range frequency sweep with (red solid line) and without (blue dashed line) parametric amplification. The value of $A_{d}$ was set to $0.05 \mathrm{~V}$, while the value of $A_{p}$ was set to $14.25 \mathrm{~V}$ to give maximum amplification for $M_{2}$ without inducing any instabilities. 


\section{CONCLUSIONS}

In this work, we have demonstrated that parametric amplification extended to the case of multiple resonances is a simple and effectual method of controlling the effective resonant quality factor in a multi-degree of freedom resonant chemical mass sensor array. By increasing the effective quality factor, the minimum detectable mass can be reduced which leads to an increase in device sensitivity. Since the minimum detectable mass scales with the quality factor according to $Q^{-1 / 2}$, the gain in sensitivity can be expressed as $G_{s}=\sqrt{Q_{o n} / Q_{\text {off }}}$. From the experiments, the sensitivity gains for microcantilevers $M_{1}$ through $\mathrm{M}_{4}$ were calculated to be $1.63,1.41,1.61$, and 1.52 , respectively. The limitation of our technique is that a sufficiently small damping is required initially. As well, the gain in effective quality factor can also be limited by nonlinearities. Nevertheless, by supplementing the usual frequency sweep with a properly selected parametric pump one can effectively amplify the important resonances of the sensing structure. This results in higher effective quality factors in the sweep response and can be used to enhance the sensitivity of swept MEMS devices that rely on resonance location for measurement.

\section{ACKNOWLEDGEMENTS}

This work was supported by NSF Sensors and Sensor Systems Program Grants $\underline{0758419}$ and $\underline{0800753}$, and by ICB Sensors Grant W911NF-09-D-0001. The authors would like to thank Dr. Barry DeMartini for fabricating the device and providing preliminary data on the device characteristics.

\section{REFERENCES}

[1] P.S. Waggoner and H.G. Craighead, "Micro- and nanomechanical sensors for environmental, chemical, and biological detection”, Lab on a Chip, 7, 1238 (2007).

[2] A.N. Cleland, "Thermomechanical noise limits on parametric sensing with nanomechanical resonators", New Journal of Physics, 7, 235 (2005).

[3] D. Rugar and P. Grütter, "Mechanical parametric amplification and thermomechanical noise squeezing", Physical Review Letters, 67, 699 (1991).

[4] J.F. Vignola, J.A. Judge, J. Jarzynski, M. Zalalutinov, B.H. Houston, and J.W. Baldwin, "Effect of viscous loss on mechanical resonators designed for mass detection”, Applied Physics Letters, 88, 041921 (2006).
[5] T.P. Burg, A.R. Mirza, N. Milovic, C.H. Tsau, G.A. Popescu, J.S. Foster, and S.R. Manalis, "Vacuum-packaged suspended microchannel resonant mass sensor for biomolecular detection”, Journal of Microelectromechanical Systems, 15, 1466 (2006).

[6] J. Tamayo, A.D.L. Humphris, A.M. Malloy, and M.J. Miles, "Chemical sensors and biosensors in liquid environment based on microcantilevers with amplified quality factor", Ultramicroscopy, 86, 167 (2001).

[7] J. Mertz, O. Marti, and J. Mlynek, "Regulation of a microcantilever response by force feedback”, Applied Physics Letters, 62, 2344 (1993).

[8] S. Meerkov, "Principle of vibrational control: Theory and applications", IEEE Transaction on Automatic Control, 25, 755 (1980).

[9] N.J. Miller and S.W. Shaw, "Frequency sweeping with concurrent parametric amplification”, Proceedings of the $1^{\text {st }}$ ASME Dynamic systems and Control Conference, Ann Arbor, MI, 10/20-22/08, American Society of Mechanical Engineers (2008), DSC2008-118.

[10] B.E. DeMartini, J.F. Rhoads, M.A. Zielke, K.G. Owen, S.W. Shaw, and K.L. Turner, "A single input-single output coupled microresonator array for the detection and identification of multiple analytes”, Applied Physics Letters, 93, 054102 (2008).

[11] J.F. Rhoads, N.J. Miller, S.W. Shaw, and B.F. Feeny, “Mechanical domain parametric amplification”, Journal of Vibration and Acoustics, 130, 061006 (2008).

[12] N.J. Miller, "Mechanical domain parametric amplification in multiple-degree-of-freedom systems,” M.S. thesis, Michigan State University, East Lansing, MI, 2007.

[13] J.F. Rhoads and S.W. Shaw, "The Effects of nonlinearity on parametric amplifiers", Proceedings of the ASME 2008 International Design Engineering Technical Conferences \& Computers and Information in Engineering Conference, Brooklyn, NY, 08/03-06/08, American Society of Mechanical Engineers (2008), DETC2008-49594.

\section{CONTACT}

*Z. Yie, tel: +1-805-893-7849; yie@engineering.ucsb.edu 